\title{
Primary Leiomyosarcoma Of Inferior Vena Cava : An Uncommon Entity
}

\author{
Kruti Dave, Shwetang Solanki, and Ritesh Prajapati
}

\begin{abstract}
Primary vascular leiomyosarcoma is a rare tumor that arises mainly from the inferior vena cava (IVC). It is usually a slow-growing progressive tumor. It shows mainly three type of growth patterns; $62 \%$ of cases demonstrate extraluminal pattern, 5\% cases show intraluminal pattern, and 33\% extraand intraluminal growth patterns.

Index Terms - Leiomyosarcoma, Inferior Vena Cava, Multidetector computed tomography scan
\end{abstract}

\section{CASE REPORT}

History 56-year-old woman presented with pain in epigastric region, breathlessness and discomfort of abdomen. It was associated with loss of appetite since the two months. Computed Tomography (CT) Scan was advised by the consulting physician for the same.

Multidetector computed tomography scan of whole abdomen showed markedly dilated IVC with large heterogeneously enhancing mass lesion throughout its course(Fig-1). Large extraluminal extension of the mass was seen in precaval region in retroperitoneum; which was causing anterior displacement of pancreas and completely encasing the 3rd part of duodenum (Fig-2,3). Superiorly, it was seen extending into right atrium. Extra-luminal retroperitoneal component was measuring about $8.8 \times 7.9 \mathrm{~cm}$ in size.

Right and middle hepatic veins were not visualized suggesting an obstruction. Hypertrophied caudate and left lobes of liver were seen with presence of multiple small hypodense lesions in all segments of both lobes of liver suggest secondaries(Fig-4). Portal vein was normal in size and showed normal enhancement. Multiple tortuous vascular channels were present at anterior abdominal wall, bilateral renal hilum and bilateral paracolic regions suggest collaterals. Mild ascites was also present.

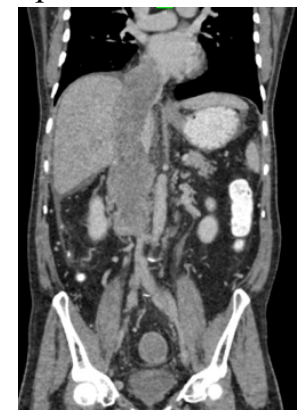

Fig. 1. CECT scan of abdomen showing markedly dilated IVC with large heterogeneously enhancing mass lesion

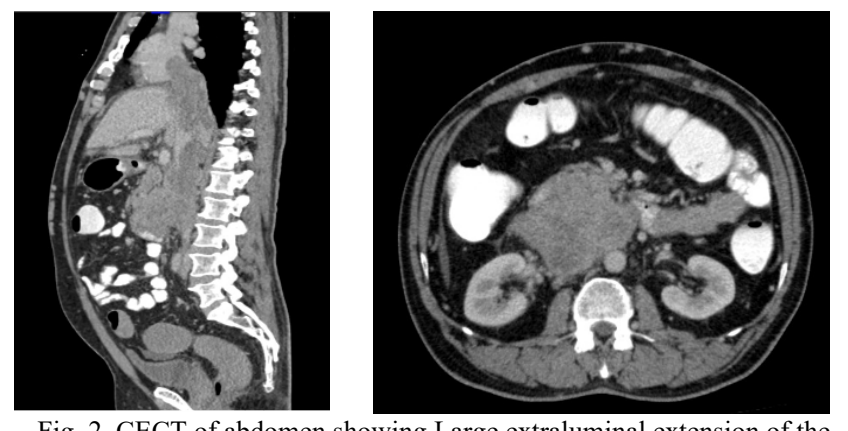

Fig. 2. CECT of abdomen showing Large extraluminal extension of the mass was seen in precaval region in retroperitoneum; which was causing anterior displacement of pancreas and completely encasing the 3rd part of duodenum.

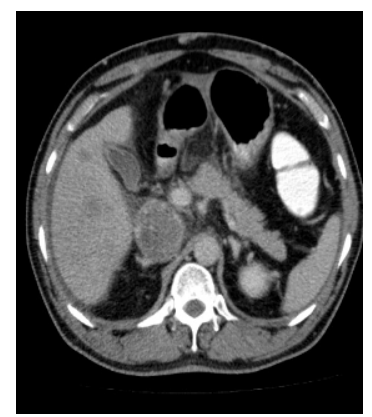

Fig. 3. CECT of abdomen showing multiple small hypodense lesions in all segments of both lobes of liver suggest secondaries

The provisional diagnosis of malignant lesion of inferior vena cava with extraluminal extension \& secondaries in liver associated with Budd Chiari syndrome was made.

The final diagnosis was made by CT guided biopsy as leiomyosarcoma of inferior vena cava. It was further proved by histopathological correlation. Final Stage

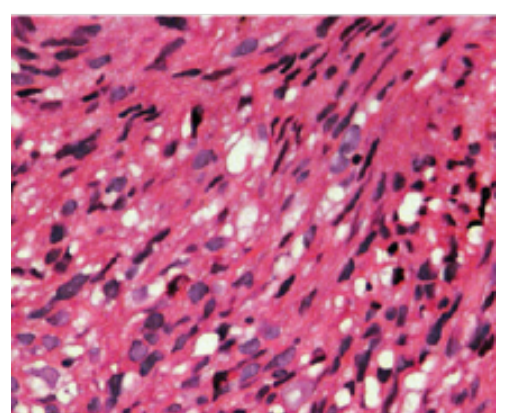

Fig. 4. Histopathology slide showed a cellular spindle cell neoplasm with interlacing bundles. The nuclei showed poly- morphism and hyperchromasia. 


\section{DISCUSSION}

Leiomyosarcoma of the inferior vena cava (IVC) is a rare malignant tumor which originates from the smooth muscle of the intima media. It is the most common primary malignancy in the IVC [1,2].

The most common location of leiomyosarcomas are mainly in the IVC, although they sometimes develop in smaller veins also. The tumor has preponderance for older women; with a male to female ratio of 1: 5[3].

For classification we can divide the IVC into three segments:

- (I) Lower segment considering below the renal veins.

- (II) Middle segment from the renal vein up to the hepatic veins.

- $\quad$ (III) Upper segment from the level of the hepatic veins to the right atrium.

Though leiomyosarcoma of the IVC most commonly occurs in the middle segment.

The tumor may solely have extrinsic or intrinsic or both the components. Tendency of tumor is to grow slowly \& expanding along the tissue planes of least resistance. Upper segment tumors give rise to varying degrees of Budd Chiari syndrome due to hepatic vein thrombosis $[4,5]$, as it was seen in our case.

In imaging findings: it appears as a lobulated retroperitoneal mass in ultrasonography. The lesion is mostly hypoechoic and can be surrounded by a hyperechoic rim. The exact location of the tumor can be seen in USG.

CT scan helps to characterize the intraluminal vascular tumor, which is usually large lobulated lesion. Sometimes it shows heterogeneousity secondary to hemorrhage and necrosis. The tumor is mainly hypovascular, but may show peripheral enhancement following contrast injection[6]. Leiomyosarcoma with extravascular component are difficult to differentiate from retroperitoneal tumors which are compressing or invading the IVC [7].

MRI shows isointense lesion on T1-weighted MR images, and iso- to hyperintense lesions on T2-weighted MR images. The area of hemorrhage appears as hyperintense on T1weighted images. MR imaging are helpful to characterize intraluminal and extraluminal tumor staging. It also helps to determines the degree involvement of adjacent organ [8].

The differential diagnosis of an intra-luminal mass in the IVC includes leiomyosarcoma, angiosarcoma and tumor thrombus [9]. The final diagnosis can be made by an ultra sound or CT guided biopsy [6].

Treatment for IVC tumor is surgical resection. Although, surgical resectability is mainly depends on the location of the tumor. Complete resection of the tumor is only possible in the lower segment. For the middle segment tumors, a more complicated en bloc resection is needed. If the renal vein is involved the resection of right kidney is usually needed. If the tumor involves the upper segment, complete resection is usually not possible due to frequent extension into the hepatic veins and the right side of the heart [10].

\section{CONCLUSION}

CT scan is the modality of choice for diagnosing IVC leiomyosarcoma. However, CT guided biopsy is always needed to confirm the diagnosis.

\section{REFERENCES}

[1] Ceyhan M, Danaci M, Elmali M, Ozmen Z. Leiomyosarcoma of the inferior vena cava. Diagn Interv Radiol 2007; 13(3):140-143.

[2] Hartman DS, Hayes WS, Choyke PL, Tibbetts GP. Leiomyosarcoma of the retroperitoneum and inferior vena cava: radiologic-pathologic correlation. RadioGraphics 1992;12(6):1203-1220.

[3] Van Rooij WJJ, Martens F, Verbeeten B, Dijkstra J. CT and MR imaging of leiomyosarcoma of the inferior vena cava. J Comput Assit Tomogr 1988; 12(3): 415-419.

[4] Mönig SP, Gawenda M, Erasmi H, Zieren J, Pichlmaier H. Diagnosis, treatment and prognosis of the leiomyosarcoma of the inferior vena cava: three cases and summary of published reports. Eur J Surg 1995;161(4):231-235.

[5] Young R, Friedman AC, Hartman DS. Computed tomography of leiomyosarcoma of the inferior vena cava. Radiology 1982;145(1):99103.

[6] Hemant D, Kranti Kumar R, Amita J, Chawla R, Ranjit N. Primary leiomyosarcoma of IVC, a rare entity: Imaging features. Australasian Radiol 2001; Nov 45(4): 448-451.

[7] Skooj SJ, Mcleod DG, Stutzman RE, Bloom DA. Leiomyosarcoma of the inferior vena cava presenting as suprarenal mass. J Urol 1983; 130: 760-772.

[8] Blum U, Wildanger G, Windfuhr M, Laubenberger J, Freudenberg N, Munzar T. Preoperative CT and MR imaging of inferior vena cava leiomyosarcoma. Eur J Radiol 1995;20(1):23-27.

[9] Gay SB, Sistronm CL, Pevarski DJ, Feldman PS. Inferior vena cava mass and Budd Chiari syndrome. Investigative Radiology 1993; 28(8): 774-776.

[10] Monig SP, Gawenda M, Erasmi H, Zieren J, Pichlmaier H. Diagnosis treatment and prognosis of leiomyosarcoma of the inferior vena cava. Eur J Surg 1995; 161: 231-235. 